\title{
Nutrition Treatment for HIV Wasting: A Prescription for Food as Medicine
}

Ann Yelmokas McDermott, PhD, LN; Abby Shevitz, MD, MPH; Aviva Must, PhD; Susan Harris, DSc; Ronenn Roubenoff, MD, MHS; Sherwood Gorbach, MD

Tufts University, Boston, MA

\begin{abstract}
Background: The optimal nutrition approach for the promotion of weight gain in HIV-infected adults with wasting remains unclear. Previous dietary interventions report minimal success and provide inadequate information regarding the counseling approach, contact time, session format, and issues addressed with the subject. The methods we report were incorporated in a 12-week intervention trial for the reversal of HIV-wasting.

Methods: All subjects involved in the intervention trial for the reversal of HIV-wasting received weekly, customized, one-on-one counseling and an oral nutrition supplement (480 kcal/d with $30 \mathrm{~g}$ protein). The nutrition aims were to (1) increase caloric intake to surpass daily energy requirements by $500 \mathrm{kcal} / \mathrm{d}$ (suggested caloric intake: 40 to $50 \mathrm{kcal} / \mathrm{kg}$ current weight); (2) increase protein intake (1.6 to $1.8 \mathrm{~g} / \mathrm{kg}$ current weight per day); and (3) identify foods that may exacerbate or curtail side effects associated with HIV. Also assessed were preconceptions, nutrition knowledge level and primary information source, and obstacles to healthy eating. Sessions, conducted by a nutritionist in an interactive, action-oriented learning approach, ranged from 30 to 60 minutes.

Results: At baseline, subjects harbored many misconceptions, reported numerous HIV-related side effects, and lacked practical nutrition strategies, all of which interfered with weight maintenance and health. The protocol strategies were acceptable to the patients (87\% subjects completed all visits), with marked improvements in dietary intake, weight, and body composition, both during and after intervention. Conclusions: We describe a customized nutrition intervention that produces changes in energy intake, maintenance of appropriate protein intake, and the reversal of unintentional weight loss over 5 to 15 months. Sustained improvements occurred across a socioeconomically diverse population, despite persistent disease-and medication-associated side effects.
\end{abstract}




\section{Introduction}

Acquired immunodeficiency syndrome (AIDS) wasting, marked by unintentional weight loss of both fat mass and lean body mass (LBM) remains a persistent and prevalent problem for human immunodeficiency virus (HIV)-seropositive adults despite the advances made in highly active antiretroviral therapy (HAART). ${ }^{1}$ Although the medical literature advocates an early and aggressive approach to nutrition support for HIV-infected individuals, information regarding the optimal treatment format and the expected outcome is lacking. Given the changing face of HIV, with minorities and women increasingly affected, it is important to define an effective nutrition intervention for a socioeconomically, racially, and ethnically diverse population. This article describes the methods used and obstacles addressed in a highly successful nutrition-based intervention designed to reverse HIV-associated wasting and conducted in a diverse, urban population of men and women.

\section{Defining HIV-Associated Wasting}

HAART, now a commonly prescribed treatment for HIV, results in improved immune function, reduced occurrence of many secondary infections, and decreased mortality. ${ }^{2}$ However, these beneficial effects are often accompanied by malabsorption, side effects such as diarrhea, nausea, and lack of appetite, and restrictive eating schedules. ${ }^{3}$ Wasting is prevalent. One third of the subjects in the Nutrition for Healthy Living Study (NFHL) at Tufts University, a longitudinal cohort study of 633 HIV-infected adults that began in February 1995, developed documented wasting ( $n=157$ of 466 included in that analysis), even though $91(58 \%)$ of 157 subjects reported taking HAART. ${ }^{1}$ Unintentional weight loss in HIV-infected adults is episodic, frequent, and associated with opportunistic infections, 4 a pattern that was evident in the NFHL study.

Wasting causes disability and death, ${ }^{5-8}$ suggesting the importance of early intervention. A number of therapeutic options have been proposed, among them nutrition intervention. However, in practice, early stages of wasting often go undiagnosed, due in part to the lack of a standardized definition of wasting. ${ }^{9}$ In our studies, ${ }^{1,10}$ we define wasting as unintentional loss of body weight of $>10 \%$ from premorbid weight, loss of $>5 \%$ in the previous 6 months, or a body mass index (BMI) $<20 \mathrm{~kg} / \mathrm{m}^{2}$. Also, our experience with medical records obtained from area clinics indicates that weight is often measured with clothes, shoes, and pocket items, and may not allow true weight change to be distinguished from differences in seasonal attire.

The course of treatment requires a complex decision process when wasting is recognized, with no one treatment strategy defined as the standard or optimal approach. ${ }^{11}$ Although a number of dietary approaches have been tested, study times have been short, results have varied, and the actual interventions have been presented only as brief descriptions, with too few details to allow for replication of the intervention. ${ }^{12-15}$ The long-term impact of nutrition intervention remains unclear. Historically, most nutrition interventions have been conducted before the common use of HAART regimens and were tested in white, middle-class, homosexual men. Success has been variable. The applicability to minorities, women, and low socioeconomic groups and the expected outcome are unknown. 
Any nutrition intervention as a treatment option for HIV-associated wasting must encompass far more than specification of the number of calories or grams of protein required per day. This is especially true given the common fluctuations in health experienced by individuals in this population. It remains a common belief that nutrition support alone is an ineffective treatment for wasting. ${ }^{16-18}$ Yet, our experience indicates that an aggressive, individualized intervention that promotes daily optimal energy and protein intake in conjunction with the management of side effects is feasible and effective. Also, weekly sessions characterized by an active-learning patient education format are able to successfully engage men and women of all socioeconomic strata. Furthermore, the nutrition intervention is beneficial, regardless of adjunct therapies, and remains so, even after intervention completion. Summarized subject information and study results from this nutrition intervention are described in an abstract. ${ }^{10}$ The goal of this paper was to describe the methods in sufficient detail for practitioners to be able to adopt them for use in their work with these populations.

\section{Effective Dietary Intervention for HIV Wasting}

\section{Study Protocol}

The primary objective of this study and analysis was to determine the effectiveness of intensive dietary counseling plus an oral nutrition supplement on energy and protein intake, weight, and fat-free mass (FFM) in HIV-seropositive adults with wasting. We compared HIV-infected persons with

documented wasting ( $>10 \%$ previous year, $>5 \%$ in past 6 months, or BMI $<20 \mathrm{~kg} / \mathrm{m}^{2}$ ) who did not receive dietary intervention ( $\mathrm{n}=56 ; 31$ men and 25 women) to participants in a 3-month clinical trial that included nutrition intervention ( $\mathrm{n}=39 ; 28$ men and 11 women). All intervention subjects received weekly, individual dietary counseling and oral liquid supplements (480 kcal/d and $30 \mathrm{~g}$ protein/d) for 3 months. Both groups met study criteria, and had identical evaluations, 3-day food records, bioimpedance analysis (BIA), and anthropometry. All 95 subjects and primary care teams received periodic status reports from assessment visits.

The goal of the nutrition intervention was to implement a dietary protocol, which would aid in the reversal of HIV-associated weight loss. The nutritional aims were to (1) increase caloric intake to surpass daily energy requirements by $500 \mathrm{kcal} / \mathrm{d}$, with the suggested caloric intake of 40 to $50 \mathrm{kcal} / \mathrm{kg}$ current body weight; (2) increase protein intake to 1.6 to $1.8 \mathrm{~g} / \mathrm{kg}$ of current body weight per day (double the recommended daily allowances); and (3) identify foods that may exacerbate or curtail symptoms and side effects associated with HIV. The primary objective of the first 6 of the 12 sessions was to increase energy and protein intake and to develop and reinforce strategies for maintaining intake in a variety of circumstances. The second 6 sessions focused on maintaining these skills while improving dietary quality. As a tool to achieve these goals, subjects were offered weekly, individual, customized dietary counseling appointments consisting of 30 to 60 minutes with a nutritionist. Eighty-seven percent of subjects completed all sessions, either in person, or if ill, by phone. Appointments were held on an outpatient basis at the General Clinical Research Center at New England Medical Center. In addition to weekly dietary assessment, information pertaining to health status, medications, medical support, and obstacles to nutrient intake were probed. A high-protein, liquid supplement (Boost High Protein, 8 fluid oz, 240 total kcal; 15 g protein; $33 \mathrm{~g}$ carbohydrate, $6 \mathrm{~g}$ fat per can, plus varying amounts of micronutrients in a lactosefree, gluten-free formula; Mead Johnson Nutritionals Research Center, Evansville, IN) was also provided with instructions to consume 2 cans/d for 12 weeks as a supplement to food intake. 
The study objective was to create a sustainable, healthful diet for weight gain in the face of entry diets that were poor in dietary quality (high in fat and low in vegetable, fruit, and fiber intake) and in the common presence of metabolic complications (fat redistribution, elevated fasting glucose, triglycerides, and cholesterol) and symptoms (nausea, diarrhea, and lack of appetite). For dietary carbohydrate and fat goals, the nutritionists followed the USDA tional emphasis on soluble and insoluble fiber.

\section{Dietary intake assessment}

Dietary information was collected by 2 methods: (1) formal assessments at baseline, midstudy, study completion (week 12), and poststudy visits based on 3-day food records and (2) a 24-hour dietary recall collected weekly.

Three-day food record instructions to record intake on 3 consecutive days ( 2 weekdays and 1 weekend day) were given in person to provide quantitative data. Food scales were distributed at the initial visit, but few subjects reported using them. Once completed, dietary records were reviewed at the next visit by the nutritionist and the subject, with the use of visual aids to ensure accurate intake data. We stressed accuracy in recording, regardless of how "good or bad" the actual quality of the diet. To address the problem of low literacy, we suggested the participants record figures or pictures as reminders of dietary intake or save food package labels. To encourage thorough and accurate records, we promised a "guiltfree" review of the diet intake record. Because of these efforts, subjects were very willing to comply with our requests ( $90 \%$ of subjects completed 3 -day food records), and we believe they delivered accurate records. If a 3-day food record was not completed for formal assessment visits, one 24-hour recall for the previous 24 hours was collected in person.

The 24-hour dietary recall collected at weekly visits was meant to provide qualitative, rather than quantitative, nutrition information, and insight into daily variability, eating patterns, and strategies. When subjects reported experiencing side effects, illness, or variable dietary intake, an additional 24-hour dietary recall was collected by phone during the following week.

All food records were entered and analyzed by 1 experienced research technician using the Minnesota Nutrient Database, version NDS 2.92. The following week, subjects received a report printout and reviewed it with the nutritionist. This process served to increase the subject's knowledge base on serving sizes, quantifying food volume, macronutrient and energy intake, and enhance awareness of the interaction of dietary intake and health fluctuation. In addition, this format offered the nutritionist repeated opportunities to assess detrimental or helpful eating patterns, current obstacles to intake, and the subjects' interpretation of health and nutritional status. Also probed repeatedly throughout the study was the use of "medicinal foods" and supplements, including energy and protein powders, bars, and drinks; micronutrient and herbal pills; and "holistic treatments." Results of the macro-and micronutrient analysis were mailed to the primary care physician.

Antiretroviral therapies present a daily challenge to adequate dietary intake because of restrictive eating schedules and side effects. Also, symptom and side effect scores have been linked to poor HAART

compliance, further compromising health. ${ }^{19}$ Questions were posed to determine what instructions had been given by the primary care team, whether the underlying importance of the medication-related food restrictions was understood, how the subject applied these instructions to daily living, and how often they applied these directions. 
Dietary assessment difficulties encountered in this population include the daily variability in energy intake, often the result of HIV-related symptoms or medication side effects (Table 1 and unpublished NFHL data). Weekly dietary assessment by multiple methods assured the likelihood of capturing this variability.

\section{Weekly counseling sessions}

Each appointment included assessment of body weight and a brief ( 5 to 10 minutes) review of health, diet, and medications with study personnel. Subjects volunteered personal circumstance information (personal relationships, work, alcohol abuse, and housing). The nutritionist conducted the remainder of the appointment. The role of the nutritionist was to present and clarify information, guide strategy applications, and record progress. In-person sessions that were canceled because of health or psychosocial crises were rescheduled as telephone appointments. Sessions then focused on coping strategies to promote energy intake during the crisis.

An interactive learning model was chosen to determine the subject's current knowledge and fundamental beliefs and to introduce facts and establish weekly practical "homework assignments." Encouragement was given to engage in open and honest communication and to ask questions. Nutritionists attempted to provide information in terms that the patient would understand and find personally meaningful. A format of give-and-take conversation was adopted, asking the subject to interpret and restate the information, foresee obstacles to successful coping skill assignments, and to problem-solve alternative solutions. During weekly sessions, the nutritionist would record the subject's goals, accomplishments, and difficulties. This validated the information's importance, allowed progress to be tracked at weekly meetings, and provided subject-directed benchmarks over time. With this approach, the subject became a partner in the nutrition and health assessment process, learning which details had impact on nutrition, how nutrition and health stability are intertwined, and the multitude and cumulative effect of these seemingly small details. Over time, this confidence led to more open communication. It was evident by the subjects' engagement during the sessions and by the attendance rate that the subjects enjoyed the process. Also, by maintaining detailed records, patterns became evident that may not have been noticed at a single session. These records were also used at weekly staff meetings to identify potential problems, suggestions, and strategies for the next session. We began each session by asking the subject if there were any questions, despite our preset agenda. Even subjects' unrelated questions were engaged, in the belief that this approach promoted patient involvement and reinforced skills. This tactic also provided the research team with an understanding of that individual's particular concerns.

In later sessions, we addressed the question of "whom to believe and why" in weighing information from media and other sources, to provide information sources subjects could access in the future. Subjects were introduced to ongoing credible sources aimed at the lay person (web sites such as the Tufts Nutrition Navigator and the American Dietetic Association, or newsletters from American Cancer Society, etc). Medical center and community-based programs providing access to HIV specialty nutritionists were also recommended for postintervention learning. In addition, we encouraged all subjects to think critically, to challenge any concepts we presented that conflicted with their current beliefs, to request scientific justification of new information, and to apply this approach beyond the study realm as a longterm strategy for health self-management.

Weekly appointments were scheduled at the patient's preference, either fixed or determined each week to best accommodate their needs. Compliance was high, with $87 \%$ of subjects completing all 12 sessions 
either in person or by phone. Although the study objectives were clearly defined, the study format was very flexible in design, enabling the staff to engage and achieve success with almost all participants. Most helpful was the nutrition staff's highly cooperative and fully committed approach to implementing this novel protocol.

\section{Assessing current nutrition knowledge and proposing change}

Nutrition knowledge varied widely, regardless of socioeconomic class. Although some subjects considered nutrition to be an integral component of disease management, their sources of information were often neither accurate nor beneficial. Because many subjects held nonmedical, nonscientific sources in high esteem and were reluctant to accept nutritional advice, it was crucial to determine the primary source of nutrition information and existing belief structure. In contrast, other subjects were very willing to follow all directions given by the research team. Questions were also asked regarding history with food, dietary variety, foods currently included in the diet, food perceptions (the role of food in health and illness, foods considered "good" or "bad"), normal food volumes, and any "rules of eating," ie, cues, time of day to eat, food combinations, and cultural norms.

The reasons given for insufficient food consumption to promote weight gain were varied, although some common obstacles were identified. Among these were symptoms that limited eating, such as diarrhea (59\%), lack of appetite (56\%), memory difficulties (54\%), and nausea (46\%) (Table 1). Poor dental health (not captured in our oral health questionnaires) plagued many of our participants and left them in chronic pain and unable to chew food. If symptoms were present, then situational triggers, current coping strategies, and their effectiveness were identified. For example, after determining whether nausea was a problem, a symptom trigger would be identified (example: the process of cooking or eating or specific foods, odors, seasonings, or medications). Such a symptom, before the intervention, would generally result in a decreased intake or no intake at all. During the session, the effect of this strategy on weight, and the side effect, was reviewed with the participant. Alternative health-promoting solutions proposed might include food temperature modification (colder foods are less aromatic), the preparation of food in batches that are divided, frozen in individual serving sizes, and reheated by microwave (with the additional benefits of shorter preparation time, less aroma, the ability to choose foods on the spur of the moment, and portion size control with little waste), the selection of bland foods, and identification of foods found to be well-tolerated in the past. Surprisingly, we did not find a decrease in the prevalence of symptoms as a result of the dietary intervention. However, given that subjects were able to increase dietary intake, weight, and fat free mass (FFM) despite these symptoms, coping strategies seem to have been effectively incorporated.

Although many subjects were concerned with energy intake, energy expenditure, the other side of the energy balance equation, was sometimes ignored. Relatively high energy and protein intake reported at baseline was insufficient to support working multiple jobs, manual labor, or extreme exercise habits and little sleep. Our energy balance assessment included not only energy intake, but also the energy costs associated with activity, work, exercise, and sleep. The restorative value of sleep was also addressed.

After several weeks of discussing dietary intake and expenditure habits, it was clear that a few of our subjects were experiencing undiagnosed eating disorders, including anorexia and exercise compulsion, often because of fear of fat redistribution (lipodystrophy). Neither they nor their medical team had previously identified this problem, and as a result, weight loss seemed unexplained. In response to this problem, the energy cost of activities specifictothe participant was introduced. Homework assignments would include small, attainable tasks to promote daily caloric surplus. Among these were 
napping, recording minutes per day spent in work or activity, and consumption of additional snacks to offset energy expenditure. Also, a summary of the literature regarding lipodystrophy and its association with HIV infection and medications and known and potential treatments, were presented in appropriate terms.

Finally, the concept of normal weight fluctuation was foreign to many subjects. Unaware of normal ranges in weight fluctuation, these subjects weighed themselves daily and became very concerned over trivial daily fluctuations.

\section{Strategies for optimizing energy intake}

The concept of "food as medicine" was the cornerstone throughout all sessions. We asked subjects to consider food as a valuable treatment modality compared with any classic medication; one that would need specific individualized energy and protein prescriptions, including dose and time directions for optimal benefit and one they would learn to understand and self-regulate. Also of importance was recognition that failure to manage the nutrition component of their condition would inevitably result in suboptimal health status, regardless of medication compliance or current HIV status. Subjects were often asked, "Without building blocks, how can you build?"

Instead of calorie counting, we chose to give broad guidelines to provide structure for decision making. One guideline for determining optimal dietary intake level was cognizance that continued weight loss (or current weight maintenance) was indicative of energy intake insufficient for weight gain. "If current caloric intake is still resulting in weight loss, then energy intake is too low to gain weight." When this was the case, we would examine not only the foods consumed, but the patterns in which they were eaten, the resulting side effects, and energy expenditure.

Promoting increased protein intake was introduced with the aid of the Common Protein Foods list. The list includes 40 commonly eaten animal-and plant-based foods, along with information on portion size, protein grams, total fat grams, and saturated fat grams per single serving. Instructions remind the subject: "Many fats and saturated fats are contained in protein foods. It is important to maintain your protein intake while reducing total fat and saturated fat. Try to choose proteins that have lower fat values." Initial instructions by the nutritionist directed the subject to include one food from this list at every meal or snack, regardless of the food volume consumed. Once this was done consistently, the subject was instructed how to read food labels to determine protein content in grams and directed to record daily protein gram intake for 3 days. After comfortably demonstrating these skills, the final step consisted of teaching the subject how to determine which protein sources were low or high in total and saturated fat, based on the Protein Foods list and food labels. Table 2 addresses HIV-specific dietary objectives and common subject reported practices and misconceptions that interfered with appropriate energy and macronutrient intake.

By design, it was the subject who ultimately decided what to incorporate to create an acceptable personalized strategy. Weekly, the subject was asked to identify and to verbalize the specific parameters of the homework assignment he/she would attempt over the next week. A typical plan might be "today I will buy frozen vegetables at the grocers; at lunch, I will throw some of these vegetables into my soup and put a piece of lettuce on my sandwich; at dinner, I will put at least one more tablespoon of vegetables than usual on my dinner plate or in the recipe." 
Given the tremendous fluctuations in health status in our subjects, one of the foundations of this intervention was the development of a customized "emergency plan." This plan was designed to provide strategies to deal with the "bad days" and the "good days." At baseline, our subjects tended to have some form of plan for eating on "good days," but lacked any strategy to maintain energy intake during sick bouts, which commonly lasted 3 days. At the weekly nutrition session after the first "bad bout," subjects commonly reported that no food was eaten when they felt sick. The development of "the emergency plan," with coping strategies to apply during the next sick bout, was introduced. The first step was to identify foods that need little or no energy to prepare. Second, items were to be purchased explicitly to maintain "the emergency shelf" in both the pantry and the refrigerator. Suggested items included cottage cheese; yogurt; graham crackers and peanut butter; child-sized snack packs/crackers and cheese/ puddings; tuna with a pop-top to be eaten on crackers, mixed with mayonnaise, or straight out of the can; canned soups with added frozen vegetables or canned legumes; frozen meals (with healthful brands encouraged); additional canned supplements; and powdered instant breakfast packs with milk. When the second crisis bout occurred, subjects most often reported being prepared. The coping strategy was then evaluated and either accepted or refined. Each illness bout allowed the subject to adjust and become comfortable with the process, resulting in the subjects feeling prepared and in control. Regardless of socioeconomic background and education level, most subjects lacked basic food preparation skills, were unable to read or use food label information (although some knew how to find basic caloric and fat numbers), and were unaware of food safety. Common lesson plans included basic cooking, food shopping, label reading, comparison shopping, coupon clipping, food safety, and eating away from home.

Ingestion of a wide array of micronutrient and herbal supplement formulations and doses was reported by participants at baseline. One major concern of the nutritionists was that money was being diverted from food to unproven therapies. A few participants spent more than $\$ 100$ per month on self-selected pills or powders offering no established medical benefit. Also, these subjects were unaware of potential harm from these therapies. Issues associated with over-thecounter micronutrient and herbal supplementation as they relate to HIV-infection, antiretroviral medications, manufacturing standards, and US regulations were gradually addressed. Special focus was paid to the financial implications of self-prescribing pharmacologic doses

of supplements and their secondary impact on energy intake and health. Our focus was to promote food as the vehicle for macro-and micronutrient intake and to reduce supplement use to a multivitamin, unless prescribed by their primary care physician.

\section{Strategies for incorporating liquid supplements}

The study protocol specified the inclusion of 2 canned supplements per day in addition to food. The literature indicates canned supplements are well tolerated for short periods with the exception of their potential to displace food and minimize expected energy intake gains. ${ }^{13,20,21}$ Our strategy was to develop guidelines for the short-term incorporation of the canned supplement, while teaching a reliance on whole foods for weight maintenance, with canned supplements used to surpass energy needs and promote weight gain.

The following three guidelines for liquid supplements were proposed: (1) canned supplements are always accompanied by a snack (of any size); (2) they are never taken immediately before eating a meal; and (3) the daily limit is a maximum of 2 cans. If there were difficulties either with drinking the whole can ( $8 \mathrm{oz})$ 
at once or the product itself, alternatives were offered. These included splitting the can into smaller portions or incorporating the liquid supplement into easy recipes, such as instant pudding, blending with fruits, or freezing into a popsicle. About $10 \%$ of the subjects intermittently reported bloating after consuming a whole can.

All subjects were able to meet the 2 can/d recommendation for the entire 12 weeks, including the few who reported a preference for whole foods rather than caloric supplements. However, some subjects initially wanted to take $>2$ cans/d in lieu of food, and this was especially true during days with illness or hectic schedules away from home.

\section{Adequate Dietary Intake Obstacles: Food and Non-Food Issues}

Our research team found that much of the counseling sessions was spent addressing non-food issues, which is not surprising given the complexities of living with HIV. Table 3 summarizes the major obstacles commonly reported by subjects during our study. Many of the issues we addressed were not historically in the nutritionist's purview. Furthermore, these issues contributed directly to weight loss or impeded weight gain. Given the chronic nature and varying combination of impediments, we believe identification of and potential solutions for specific barriers were mandatory for weight gain to be achievable by each individual.

In addition to being responsible for their own health and living situations, many subjects cared for ill family members, faced legal issues, and were subject to insecure housing. Food insecurity was an additional stress. These issues were diverse in nature; the nutritionist's role was limited to assisting the subject in determining the most appropriate resources. In doing so, the subject was encouraged to take an active role in his/her medical and social welfare. Our objectives were to provide nutrition knowledge and fundamental behavior skills that could be used by the patient when faced with periodic or ongoing barriers. An example of this application process would address the following problem: HIV-infected individuals often face health fluctuations. During the 3-month intervention, many subjects experienced multiple health fluctuations of varying severity, with diarrhea being particularly common. The concept of "food as medicine" was then presented as an adjunct treatment for health stabilization, creating the potential for optimal health during the intervention (short-term) and poststudy periods. The beneficial effects of sound food habits were presented in conjunction with reinforcement of medication compliance, regular primary care team contact, and their interrelationship. Through daily decisions, the subject's ability to influence their nutrient intake, immune function maintenance, prevention of secondary infections, and early detection would be an increased likelihood of stable health status and weight gain. Also, the consequence of unstable health was explained. Lack of planning, preparation, and follow through, and its resultant crisis management approach to health, inevitably lead to periods of greater energy need accompanied by lower energy intake and cause greater physical and emotional stress.

\section{Conclusion}

A customized nutrition intervention that produces changes in energy intake, maintenance of appropriate protein intake, and the reversal of unintentional weight loss over 5 to 15 months was described. Sustained improvements occurred across a socioeconomically diverse population, despite persistent disease-and medication-associated side effects. 
The intervention format consisted of weekly, customized, one-on-one counseling in a supportive environment, and an oral caloric supplement ( $480 \mathrm{kcal} / \mathrm{d}$, with 30 g protein). Sessions were conducted with a nutritionist using an interactive, action-oriented learning approach. A strong nutrition foundation that incorporated the concept of "food as medicine" was accompanied by effective behavioral strategies, with problem solving and crisis management techniques defined by and specific to the patient's needs. Twelve weekly sessions allowed sufficient time to introduce concepts, refine coping skills, and address a diverse array of issues bearing on nutritional status. Changing lifestyle habits requires commitment and substantial investment on the part of the individual and the medical team. By understanding the relationship of food choices, weight stability, and health status, our intervention fostered patient empowerment and provided an effective, sustainable treatment strategy for the reversal of HIVassociated wasting, regardless of adjunct therapies.

\section{Acknowledgments}

We thank the Nutrition for Healthy Living nutrition team and the Nutrition, Exercise Physiology and Sarcopenia Laboratory exercise physiologists for their expertise and commitment to conducting this intervention. We are grateful for the ongoing support of the clinical staff, Sally Skinner, and the data management team of the Nutrition for Healthy Living project, and the New England Medical Center General Clinical Research Center staff. This study was supported by NIDDK Grants P01-DK45734 and R01-DK51011, the General Clinical Research Center funded by the National Center for Research Resources of the NIH (Grant M0-RR00054), and Lifespan/Tufts/Brown Center for AIDS Research (Grant P03A142853). 


\section{References}

1. Wanke C, Silva M, Knox TA, et al. Weight loss and wasting remain common complications in individuals infected with human immunodeficiency virus in the era of highly active antiretroviral therapy. Clin Infect Dis 31:803-806, 2000

2. Mocroft A, Katlama C, Johnson AM, et al. AIDS across Europe, 1994-98: The EuroSIDA study. Lancet 356:291-296, 2000

3. Cunningham WE, Shapiro MF, Hays RD, et al. Constitutional symptoms and health-related quality of life in patients with symptomatic HIV disease. Am J Med 104:129 -136, 1998

4. Macallan DC, Noble C, Baldwin C, et al. Prospective analysis of patterns of weight change in stage IV human immunodeficiency virus infection. Am J Clin Nutr 58:417-424, 1993

5. Strawford A, Hellerstein M. The etiology of wasting in the human immunodeficiency virus and acquired immunodeficiency syndrome. Semin Oncol 25:76 -81, 1998

6. Ott M, Lembcke B, Fischer H, et al. Early changes of body composition in human immunodeficiency virus-infected patients: Tetrapolar body impedance analysis indicates significant malnutrition. Am J Clin Nutr 57:15-19, 1993

7. Sharpstone D, Murray C, Ross $\mathrm{H}$, et al. The influence of nutritional and metabolic status on progression from asymptomatic HIV infection to AIDS-defining diagnosis. AIDS 13:1221-1226, 1999

8. Wheeler DA, Muurahainen N, Elion R, et al. Change in body weight (wt) as a predictor of death and opportunistic complications (OC) in HIV by history of prior OC. Int Conf AIDS 11:332, 1996

9. Steinhart CR. HIV-associated wasting in the era of HAART: A practice-based approach to diagnosis and treatment. AIDS Reader 11:557-560, 2001

10. McDermott AY, Shevitz A, Roubenoff R, et al. The effect of customized, intensive dietary intervention in HIV-infected adults with wasting. Am J Clin Nutr 75:350S, 2002

11. Polsky B, Kotler D, Steinhart CR. HIV-associated wasting in the HAART era: Guidelines for assessment, diagnosis, and treatment. AIDS Patient Care Std 15:411-423, 2001

12. Rabeneck L, Palmer A, Knowles JB, et al. A randomized controlled trial evaluating nutrition counseling with or without oral supplementation in malnourished HIV-infected patients. J Am Diet Assoc 98:434-438, 1998

13. Schwenk A, Steuck H, Kremer G. Oral supplements as adjunctive treatment to nutritional counseling in malnourished HIV-infected patients: Randomized controlled trial. Clin Nutr 18:371-374, 1999

14. Hoh R, Pelfini A, Neese RA, et al. De novo lipogenesis predicts short-term body-composition response by bioelectrical impedance analysis to oral nutritional supplements in HIV-associated wasting. Am J Clin Nutr 68:1541-1563, 1998

15. Shabert JK, Winslow C, Lacey JM, et al. Glutamine-antioxidant supplementation increases body cell mass in AIDS patients with weight loss: A randomized, double-blind controlled trial. Nutrition 15:860-864, 1999

16. Kotler D. Nutritional alterations associated with HIV infection. J Acquir Immune Defic Syndr 25:S81S87, 2000 
17. Gibert CL, Wheeler DA, Collins G, et al. Randomized, controlled trial of caloric supplements in HIV infection. Terry Beirn Community Programs for Clinical Research on AIDS. J Acquir Immune Defic Syndr 22:253-259, 1999

18. Chlebowski RT, Grosvenor M, Lillington L, et al. Dietary intake and counseling, weight maintenance, and the course of HIV infection. J Am Diet Assoc 95:428 -435, 1995

19. Ammassari A, Murri R, Pezzotti P, et al. Self-reported symptoms and medication side effects influence adherence to highly active antiretroviral therapy in persons with HIV infection. J Acquir Immune Defic Syndr 25:445-449, 2001

20. Berneis K, Battegay M, Bassetti S, et al. Nutritional supplements combined with dietary counseling diminish whole body protein catabolism in HIV-infected patients. Eur J Clin Nutr 30:87-94, 2000

21. Burger B, Schwenk A, Junger H, et al. Oral supplements in HIV-infected patients with chronic wasting. A prospective trial. Med Klin 89:579 -581, 1994

22. Roberts KJ. Barriers to and facilitators of HIV-positive patients' adherence to antiretroviral treatment regimens. AIDS Patient Care Std 14:155-168, 2000 


\section{Tables}

Table 1: Prevalence of symptoms reported by HIV-infected adults with wasting in the month prior to baseline, month 3 , and post-study visits

\begin{tabular}{lccr}
\hline \multicolumn{1}{c}{ Symptom } & Baseline $(\mathrm{n}=39)$ & $\begin{array}{c}\text { Week 12 } \\
(\mathrm{n}=38)\end{array}$ & $\begin{array}{c}\text { Post-study } \\
(\mathrm{n}=37)\end{array}$ \\
\hline Diarrhea & $23(59.0)$ & $26(56.5)$ & $19(50.0)$ \\
Dry mouth & $18(46.2)$ & $16(42.1)$ & $16(43.2)$ \\
Fever & $6(15.4)$ & $8(17.4)$ & $9(23.7)$ \\
Gum pain & $3(7.7)$ & $3(7.9)$ & $6(15.8)$ \\
Lack of appetite & $22(56.4)$ & $23(60.5)$ & $17(44.7)$ \\
Poor memory & $21(53.9)$ & $20(43.5)$ & $11(29.7)$ \\
Mouth sores & $6(15.4)$ & $5(13.2)$ & $6(15.8)$ \\
Nausea & $18(46.2)$ & $15(39.5)$ & $16(42.1)$ \\
Dysphagia & $3(7.7)$ & $5(13.2)$ & $3(7.9)$ \\
Stomach pain & $14(35.9)$ & $14(36.8)$ & $7(35.1)$ \\
Thrush & $6(15.4)$ & $4(10.5)$ & $7(18.4)$ \\
\hline
\end{tabular}

All $p=$ not significant from baseline.

Numbers are shown as n (\%). 


\section{Table 2: Energy and macronutrient intake: HIV-specific counseling objectives and subject reported practices and misconceptions that prevent optimal dietary intake}

\section{Energy Intake}

\section{Nutritionist's objectives}

To assess current nutrition knowledge, information sources, and readiness to change

To determine specific foods or dietary intake patterns which may exacerbate or curtail HIV-related symptoms

To find the energy intake level that allows for weight gain

To teach eating strategies for the "bad days" as well as "good days"

Subject practices commonly reported at baseline

Wide range in baseline energy intake reported: 15 to $87 \mathrm{kcal} / \mathrm{kg} /$ day

Withholding food to prevent symptoms

Not eating food on "bad days"

Not eating in the evening after dinner

Eating high fat (especially saturated fat) meals

Choosing fried foods

Eating diets low in fruits and vegetables (except for french fries)

Heavy reliance on fast foods, full-sugared sodas, processed junk foods, hard candy

Skipping meals, especially breakfast

Subject misconceptions reported at baseline

If you're not hungry, your body doesn't need to eat

Micronutrient and herbal supplements "give you energy"

\section{Protein}

Nutritionist's objectives

To teach about food sources high in protein, low in saturated fat

Subject practices commonly reported at baseline

Wide range in baseline protein intake: 0.5 to $4.7 \mathrm{~g} / \mathrm{kg}$

Over-reliance on meats results in high total and saturated fat diet

Some individuals consume protein powders, shakes, bars

Subject misconceptions reported at baseline

Engineered protein or amino acids from supplement manufacturers are more beneficial than food sources

Plant sources of protein are ineffective

Consuming large volumes of meat per sitting is a good habit

Fast food chains offer "good," inexpensive meat products

\section{Carbohydrates}

\section{Nutritionist's objectives}

To teach the effect of soluble and insoluble fiber on HIV-related metabolic abnormalities (lipodystrophy, lipid profile, insulin resistance)

To teach the use of soluble fiber in symptom management (diarrhea)

To teach the value of complex carbohydrates as important carriers of micronutrients and phytochemicals 
To teach food preparation methods which maintain micronutrient content

Subject practices commonly reported at baseline

Throwing away canned or frozen goods from the food pantry or meals services

Eating only fresh fruits and vegetables (no canned or frozen) results in eating few if any fruits and vegetables

Subject misconceptions reported at baseline

Fruits and vegetables have fewer calories, therefore they are not valuable for health and weight gain

Fruits and vegetables will give me diarrhea, gas, indigestion

"They don't taste good"; "I don't like any of them"; "I have never tried that kind before" Fruits and vegetables have to be fresh to be good; canned or frozen products "are no good" or "have no nutrients left"

Fruits and vegetables are too expensive and have too short a shelf life

Difficult (don't know how) to prepare vegetables

\section{Fats}

Nutritionist's objectives

To teach the physiologic effects of dietary fat volume and type of dietary fat on HIV-related metabolic abnormalities (central adiposity, lipid profile, cardiovascular risk)

To teach the effect of fat on HIV-related malabsorption symptoms (diarrhea)

Subject practices commonly reported at baseline

Dietary fat intake contributing too great a percentage of daily energy

Consciously incorporating fats in meals: the message from everywhere has been to add fats to everything in order to boost caloric intake... "good for you"

Subject practices commonly reported at baseline

Heavy reliance on fast foods and processed meats (bologna, hot dogs)

Choosing full-fat dairy products, fried foods, using butter for flavoring foods Afraid to reduce dietary fat intake, afraid will lose more weight

Subject misconceptions reported at baseline

High-fat foods are an easy, beneficial way to increase caloric intake (bacon, butter, cream, full-fat ice cream, whole milk, cream cheese)

\section{Snack Foods}

Nutritionist's objectives

To teach the concept of "empty calories" and identify "empty calories" foods

To identify flavorful, nutrient-rich snack food choices

Subject practices commonly reported at baseline

Heavy intake of hard candy, candy bars, soda, processed packaged cakes and cookies (Little Debby® cakes), ice cream contributing too great a percentage of energy intake

Junk food displacing high quality foods and further reducing appetite

Subject misconceptions reported at baseline

Snacking is "bad"

High-fat content foods are good for weight gain 


\section{Alcohol}

\section{Nutritionist's objectives}

Reduce intake in drinkers to 2 drinks per day

To educate about alcohol's effect on food displacement, appetite, liver function and metabolic issues in HIV-infected persons

Subject practices commonly reported at baseline

While many subjects report abstaining from alcohol and illicit drug use, some report regular use Binge-drinking problematic for some; may not be captured in 3-day food or 24-hour recall assessments Available money spent on alcohol and illicit drugs contributing to financial difficulties, which limit food purchase

Alcohol displacing food

Subject misconceptions reported at baseline

Binge drinking not problematic if you can continue to function during the days

A few drinks each day presents no problem in HIV-infected adults 


\section{Table 3: Obstacles to appropriate nutrient intake in HIV-infected adults with wasting}

\section{Depression}

Lack energy to shop, prepare food, clean-up

Lack interest in eating Don't want to eat alone

No appetite

Alcohol or illicit drug use

\section{Oral health}

Missing teeth; ill-fitting dentures

Extensive time between appointments to adjust new or ill-fitting dentures

Inflamed gums; abscessed teeth

Mouth sores

Poor oral hygiene practices

Thrush: undiagnosed or untreated

Inability to properly chew foods

Lack continuity of care; lack primary care dentist; rotated service providers

Treatment by emergency room dentists

Minimal standard of care provided by the public healthcare system

Dental team not an integral part of the HIV-medical team

Regular maintenance appointments not scheduled or attended "Crisis management" approach to oral health

\section{Family/social support}

Lack contact with family or friends

Isolation

Subject as parent/care-giver for others

Social support person is also ill HIV-status not disclosed to others

\section{Nutrition beliefs/misinformation}

Lack nutrient density info for specific foods

Vitamin pills, like food, can provide needed energy

Vegetables and fruit are not good for people with diarrhea

High-fat foods are good to eat often if you are losing weight

Inadvertent use of supplements which promote diarrhea ("cleansing the system of toxins")

Because water has no calories, it is not important

\section{Housing and finance}

Lack adequate kitchen facilities

Temporary sheltered housing

Finances affect food selection and/or purchase

Lack transportation to shopping Available prepared meals inappropriate for needs

\section{Work, exercise}

Difficulty having readily available food (poor planning, no work break or storage place)

Health status not disclosed at work

High-energy expenditure, little sleep

\section{HIV-infection or medication effects}

Diarrhea

Malabsorption

Lack of appetite; nausea

Dry mouth

Stomach pain 
Memory difficulties

Medications define eating schedules

Medications dictate food to be eaten

Withholding food or water to prevent or control diarrhea

Neuropathy preventing shopping and cooking

\section{Food preparation skills}

Lack cooking skills

Unable to read or interpret food labels, recipes

Monotonous, inappropriate diets

Poor food safety 